Revista de Matemática: Teoría y Aplicaciones 1999 6(2) : 97-106

CIMPA - UCR - CCSS ISSN: 1409-2433

\title{
EL ESPACIO COCIENTE Y ALGUNAS PROPIEDADES GEOMÉTRICAS DE LOS ESPACIOS DE BANACH*
}

\author{
José R. Morales ${ }^{\dagger}$
}

Recibido: 18 noviembre 1998

\begin{abstract}
Resumen
En esta nota enunciaremos algunas propiedades geométricas de los espacios de Banach, entre las cuales podemos señalar espacios uniformemente convexos, espacios uniformemente no cuadrados, espacios localmente uniformemente convexos, espacios estrictamente convexos, etc., y analizaremos el problema cuando tales propiedades se trasladan al espacio cociente.
\end{abstract}

Palabras Clave: Espacio uniformemente convexo, espacio uniformemente no-cuadrado, espacio LUR, espacio (R).

\section{Resumen}

We state some geometric properties of Banach spaces, such as uniformly convex spaces, uniformly non-square spaces, local-uniformly convex spaces, strictly convex spaces, etc., and we analize the problem of translating such properties to the quotient space.

Keywords: uniformly convex space, uniformly non-square space, LUR space, (R) space.

AMS Subject Classification: 46B20, 46B10.

\section{Notación}

Seguiremos la terminología estandar que puede encontrarse en [2]. Por $E$ denotaremos el espacio de Banach $(E,\|\cdot\|)$. $B_{E}$, denota la bola unitaria cerrada de $E, S_{E}$, la esfera unitaria de $E$ y $E^{*}$ el dual topológico de $E$.

\footnotetext{
* Este trabajo fue financiado por CDCHT-ULA: C-903-98-05-B

† Grupo de Análisis Funcional, Departamento de Matemáticas, Facultad de Ciencias, Universidad de los Andes, Mérida, Venezuela; E-Mail: moralesj@ciens.ula.ve
} 


\section{Preliminares}

Sea $E$ un espacio de Banach y $F \subseteq E$ un subespacio cerrado. Entonces, el espacio cociente $E / F$ es el espacio cuyos elementos son traslaciones de $F$, con la norma:

$$
\|x+F\|=\|\tilde{x}\|=\inf \{\|x+y\| / y \in F\} .
$$

La función

$$
\begin{aligned}
\pi: & E \rightarrow E / F \\
& x \rightarrow x+F
\end{aligned}
$$

es llamada la proyección canónica de $E$ sobre $E / F$.

Es importante señalar que la norma de $\tilde{x}$ es exactamente $d(x, F)$, donde $d(x, F)$ es la distancia desde $x$ al subconjunto $F$.

Definición 1 Sea $E$ un espacio de Banach. Un subespacio $F$ de E se llama proximinal si $\forall x \in E$, existe $y \in F$ tal que $d(x, F)=\|x-y\|$.

En [13] se prueba que un espacio de Banach $E$ es reflexivo si y sólo si cada subespacio de $E$ es proximinal. El siguiente hecho (ver [12]) es fundamental en la prueba de nuestros resultados. Sea $E$ un espacio de Banach, un subespacio cerrado $F$ de $E$ es proximinal si y sólo si la imagen de la bola unitaria cerrada de $E$ por la aplicación cociente, $\pi: E \rightarrow E / F$, es la bola unitaria cerrada de $E / F$.

\section{Definiciones}

En esta sección nos dedicaremos a enunciar aquellas propiedades geométricas de los espacios de Banach en las cuales estamos interesados.

En 1936, J.A. Clarkson [1] introdujo los espacios uniformemente convexos en la forma siguiente.

Definición 2 Un espacio de Banach $E$ es uniformemente convexo, denotado (UR), si dado $\epsilon>0$ existe $\delta=\delta(\epsilon)>0$ tal que para todo $x, y \in B_{E} y\|x-y\| \geq \epsilon$ entonces $\left\|\frac{x+y}{2}\right\| \leq 1-\delta$.

En términos de sucesiones la anterior definición puede escribirse como: un espacio de Banach $E$ es $(U R)$ si y sólo si $\left(x_{n}\right),\left(y_{n}\right) \subset B_{E}$ y $\lim _{n \rightarrow \infty}\left\|x_{n}+y_{n}\right\|=2$ entonces $\lim _{n \rightarrow \infty}\left\|x_{n}-y_{n}\right\|=0$.

D.P. Milman en 1938 y B.S. Pettis en 1939 (ver [6]), de manera independiente demostraron que si $E$ es un espacio de Banach $(U R)$ entonces $E$ es un espacio reflexivo.

En 1964, R.C. James [8] introdujo otro tipo de espacios, llamados los espacios uniformemente no-cuadrados. 
Definición 3 Un espacio de Banach $E$ se dice que es uniformemente no-cuadrado si y sólo si existe $\delta>0$ tal que si

$$
x, y \in B_{E}, \quad\left\|\frac{x+y}{2}\right\|>1-\delta \Rightarrow\left\|\frac{x-y}{2}\right\|<1-\delta .
$$

Es claro que $(U R)$ implica uniformemente no-cuadrado. James en el mismo artículo mostró que si $E$ es un espacio de Banach uniformemente no-cuadrado entonces $E$ es reflexivo.

En 1955, K. Fan y I. Glicksberg [3] generalizaron los espacios (UR) e introdujeron los espacios $(k R)$.

Definición 4 Sea $k \geq 2$ un entero. Un espacio de Banach $E$ se dice $k R$ si para toda sucesión $\left(x_{n}\right) \subset$ E tal que si

$$
\lim _{n_{1}, \cdots, n_{k} \rightarrow \infty}\left\|x_{n_{1}}+\cdots+x_{n_{k}}\right\|=k
$$

entonces $\left(x_{n}\right)$ es una sucesión de Cauchy en E.

En el mismo artículo los autores mostraron la serie de implicaciones

$$
(U R) \Rightarrow 2 R \Rightarrow \cdots \Rightarrow k R \Rightarrow(k+1) R \Rightarrow \text { reflexividad. }
$$

En 1991, B.-L. Lin y W. Zhang [9] generalizaron los espacios $k R$ y definieron los espacios $w R$.

Definición 5 Sea $E$ un espacio de Banach. Se dice que $E$ es un espacio $w R$ si para cualquier sucesión $\left(x_{n}\right) \subset B_{E}$ con

$$
\forall k \in \mathbb{N}: \quad \lim _{n_{1} \cdots n_{k} \rightarrow \infty}\left\|x_{n_{1}}+\cdots+x_{n_{k}}\right\|=k
$$

entonces $\left(x_{n}\right)$ es convergente en $E$.

Es claro que, para todo $k \in \mathbb{N}, k R$ implica $w R$ y además los autores antes mencionados probaron que si $E$ es un espacio $w R$ entonces $E$ es reflexivo.

En 1955, A.R. Lovaglia [10] localiza los espacios (UR), en la forma siguiente.

Definición 6 Un espacio de Banach $E$ es localmente uniformemente convexo, denotado (LUR), si dado $\epsilon>0$ y $x \in S_{E}$ existe $\delta=\delta(\epsilon, x)>0$ tal que

$$
\forall y \in B_{E} y\|x-y\| \geq \epsilon \Rightarrow\left\|\frac{x+y}{2}\right\| \leq 1-\delta .
$$

En términos secuenciales la anterior definición puede escribirse como: un espacio de Banach $E$ es $(L U R) \Leftrightarrow(x) \in S_{E}, \quad\left(x_{n}\right) \subset B_{E} \lim _{n \rightarrow \infty}\left\|x+x_{n}\right\|=2$ entonces $\lim _{n \rightarrow \infty}\left\|x_{n}-x\right\|=0$. 
Es claro que $(U R)$ implica $(L U R)$. Los espacios $(L U R)$ no implican la reflexividad del espacio (ver [7],[10]).

En 1988, N. Chao-Xun y W. Jian-Hua [15] localizaron los espacios $k R$ e introducen los espacios $L k R$, y en 1991 en [9] los autores definieron los espacio $L w R$. Es importante señalar que los espacios $L k R$ y los espacios $L w R$ también pueden ser vistos como una generalización de los espacios $(L U R)$.

Definición 7 Sea $k \geq 1$ un entero. Un espacio de Banach $E$ se dice LkR si para todo $x \in S_{E}$ tal que

$$
\lim _{n_{1} \cdots n_{k} \rightarrow \infty}\left\|x+x_{n_{1}}+\cdots+x_{n_{k}}\right\|=k+1
$$

entonces

$$
\lim _{n \rightarrow \infty}\left\|x_{n}-x\right\|=0
$$

Definición 8 Un espacio de Banach $E$ se dice LwR si para todo $x \in S_{E}$ y toda sucesión $\left(x_{n}\right) \subset B_{E}$ tal que

$$
\lim _{n_{1} \cdots n_{k} \rightarrow \infty}\left\|x+\sum_{i=1}^{k} x_{n_{i}}\right\|=k+1, \quad \forall k \in \mathbb{N}
$$

entonces

$$
\lim _{n \rightarrow \infty}\left\|x_{n}-x\right\|=0
$$

Es claro que, $(L U R) \Leftrightarrow L 1 R \Rightarrow \cdots \Rightarrow L k R \Rightarrow L(k+1) R \Rightarrow L w R$.

Sobre los espacios estrictamente convexos, su paternidad no es muy clara y la referencia más antigua aparece en el trabajo de Clarkson [1] pero en la monografía de V.I. Istratescu [7] se dice que tales espacios fueron definidos de manera independiente por Clarkson y M.G. Krein.

Definición 9 Un espacio de Banach $E$ se dice estrictamente convexo (R) si para todo $x, y \in S_{E} \quad y\|x+y\|=2 \Rightarrow x=y$.

Es claro que $L w R$ implica $R$.

En 1959, K. Fan y I. Glicksberg [4] introdujeron la propiedad $(H)$ en espacios de Banach $(R)$ y es M.M. Day [2] quien le elimina la condición de ser el espacio estrictamente convexo.

Definición 10 Un espacio de Banach $E$ se dice que posee la propiedad $(H)$ si para todo $x \in S_{E} \quad y \quad\left(x_{n}\right) \subset S_{E}$ con $x_{n} \stackrel{w}{\rightarrow} x$ entonces

$$
\lim _{n \rightarrow \infty}\left\|x_{n}-x\right\|=0
$$

Claramente se tiene que $L w R$ implica la propiedad $(H)$.

En 1980, R. Huff [6] generalizó los espacios $(U R)$ y en términos secuenciales introdujo los espacios casi uniformemente convexos y los espacios uniformemente Kadec-Klee, además de dar una reformulación de la propiedad $(H)$. 
Definición 11 Sea E un espacio de Banach. Se dice que E es uniformemente KadecKlee, denotado $(U k k)$, si para todo $\epsilon>0$ existe $0<\delta<1$ tal que

$$
\left(x_{n}\right) \subset B_{E} \quad y \quad x_{n} \stackrel{w}{\longrightarrow} x \quad y \quad \operatorname{Sep}\left(x_{n}\right) \geq \epsilon
$$

entonces

$$
\|x\|<\delta
$$

Definición 12 Un espacio de Banach $E$ se dice casi uniformemente convexo, denotado $(N U C)$, si para todo $\epsilon>0$ existe $0<\delta<1$ tal que $\left(x_{n}\right) \subset B_{E}$ y $\operatorname{Sep}\left(x_{n}\right) \geq \epsilon$ entonces

$$
C_{0}\left(x_{n}\right) \cap B_{\delta}(U) \neq \emptyset .
$$

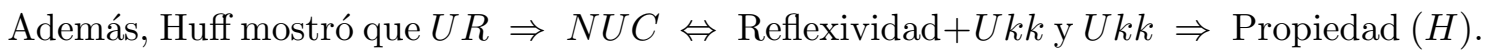

\section{Resultados}

En esta sección consideramos el problema cuando una de las propiedades del espacio $E$, enunciadas en la sección anterior, son transmitidas al subespacio cociente.

Generalmente, los espacios cociente no conservan estas propiedades, como lo podemos ver en el ejemplo dado por Klee que aparece en [7] donde se muestra que la propiedad de ser $E$ un espacio $(R)$ no se traslada al espacio cociente. Sin embargo, bajo ciertas condiciones la heredabilidad se cumple.

El siguiente resultado nos sintetiza algunas propiedades transmitidas al cociente.

Teorema: 1 Sea $E$ un espacio de Banach y $F \subseteq E$ un subespacio cerrado de E. Se tiene que:

a) Si E es (UR) entonces E/F también lo es.

b) Si E es reflexivo y (LUR) entonces $E / F$ es (LUR).

c) Sea $E$ (LUR). Si F es un subespacio proximinal de $E$ entonces $E / F$ es (LUR).

d) Si $E$ es $(R)$ y $F$ es un subespacio reflexivo entonces $E / F$ es $(R)$.

Prueba: a) ver Intratescu [7].

b) y c) ver Montesinos \& Torregrosa [12].

d) se debe a Klee, ver [7].

Teorema: 2 Sea $E$ un espacio de Banach uniformemente no-cuadrado y $F \subseteq E$ un subespacio cerrado de $E$. Entonces, $E / F$ es un espacio uniformemente no-cuadrado.

Prueba: Supongamos que existe $\delta>0$.

Sean $\tilde{x}, \tilde{y} \in B_{E / F}$ tales que $\left\|\frac{\tilde{x}+\tilde{y}}{2}\right\|>1-\delta$. Queremos mostrar que $\left\|\frac{\tilde{x}-\tilde{y}}{2}\right\| \leq 1-\delta$.

Como $E$ es reflexivo, entonces $S_{E / F} \subset \pi\left(S_{E}\right)$, por tanto existen $x, y \in B_{E}$ tales que

$$
\pi(x)=\tilde{x} \quad \mathrm{y} \quad \pi(y)=\tilde{y} .
$$


Puesto que,

$$
\left\|\frac{x+y}{2}\right\| \geq\left\|\frac{\tilde{x}+\tilde{y}}{2}\right\|>1-\delta
$$

y usando el hecho que $E$ es un espacio uniformemente no-cuadrado se tiene que

$$
\left\|\frac{x-y}{2}\right\| \leq 1-\delta
$$

Por tanto,

$$
\left\|\frac{\tilde{x}-\tilde{y}}{2}\right\| \leq\left\|\frac{x-y}{2}\right\| \leq 1-\delta
$$

Así pues $E / F$ es un espacio uniformemente no-cuadrado.

Teorema: 3 Sea $k \geq 2$ un entero. Sea $E$ un espacio de Banach $k R$ y $F \subseteq E$ un subespacio cerrado de E. Entonces, $E / F$ es un espacio $k R$.

Prueba: Sea $(\tilde{x})$ una sucesión de $B_{E / F}$, tal que

$$
\lim _{n_{1} \cdots n_{k} \rightarrow \infty}\left\|\tilde{x}_{n_{1}}+\cdots+\tilde{x}_{n_{k}}\right\|=k .
$$

Por un argumento similar al teorema anterior existe $x_{n_{i}} \in B_{E} \quad i=1 \ldots k$ tal que $\pi\left(x_{n_{i}}\right)=\tilde{x}_{n_{i}}, \quad i=1 \ldots k$.

Ahora,

$$
\begin{aligned}
k=\lim _{n_{1} \cdots n_{k} \rightarrow \infty}\left\|\tilde{x}_{n_{1}}+\cdots+\tilde{x}_{n_{k}}\right\| & \leq \lim _{n_{1} \cdots n_{k} \rightarrow \infty}\left\|x_{n_{1}}+\cdots+x_{n_{k}}\right\| \\
& \leq \lim _{n_{1} \cdots n_{k} \rightarrow \infty}\left(\left\|x_{n_{1}}\right\|+\ldots+\left\|x_{n_{k}}\right\|\right) \\
& =k .
\end{aligned}
$$

Así,

$$
\lim _{n_{1} \cdots n_{k} \rightarrow \infty}\left\|x_{n_{1}}+\cdots+x_{n_{k}}\right\|=k .
$$

Ahora, usando el hecho que $E$ es un espacio $k R$ se tiene que $\left(x_{n}\right)$ es una sucesión de Cauchy en $E$, y como

$$
\left\|\tilde{x}_{n}-\tilde{x}_{m}\right\| \leq\left\|x_{n}-x_{m}\right\|
$$

se concluye que $\left(\tilde{x}_{n}\right)$ es Cauchy en $E / F$. Por tanto, hemos probado que $E / F$ es un espacio $k R$.

En forma similar se prueba el siguiente resultado.

Teorema: 4 Sea $E$ un espacio de Banach $w R$ y $F \subseteq E$ un subespacio cerrado de $E$. Entonces, $E / F$ es un espacio $w R$.

Teorema: 5 Sea $k \geq 1$ un entero. Sea $E$ un espacio de Banach reflexivo y LkR y $F \subseteq E$ un subespacio cerrado de E. Entonces $E / F$ es un espacio LkR. 
Prueba: Sean $\tilde{x}_{0} \in B_{E / F}$ y $\left(\tilde{x}_{n}\right) \subset B_{E / F}$ tales que,

$$
\lim _{n_{1} \cdots n_{k} \rightarrow \infty}\left\|\tilde{x}_{0}+\sum_{i=1}^{k} \tilde{x}_{n_{0}}\right\|=k+1 .
$$

existen $x_{0} \in S_{E}$ y $x_{n_{i}} \in B_{E}, \quad i=1 \ldots k$ tales que

$$
\pi\left(x_{0}\right)=\tilde{x}_{0} \quad \mathrm{y} \quad \pi\left(x_{n_{i}}\right)=\tilde{x}_{n_{i}}, \quad i=1 \ldots k .
$$

Así,

$$
\begin{aligned}
k+1 & =\lim _{n_{1} \ldots n_{k} \rightarrow \infty}\left\|\tilde{x}_{0}+\sum_{i=1}^{k} \tilde{x}_{n_{i}}\right\| \\
& \leq \lim _{n_{1} \ldots n_{k} \rightarrow \infty}\left\|x_{0}+\sum_{i=1}^{k} x_{n_{i}}\right\| \\
& \leq \lim _{n_{1} \ldots n_{k} \rightarrow \infty}\left(\left\|x_{0}\right\|+\sum_{i=1}^{k}\left\|x_{n_{i}}\right\|\right)=k+1,
\end{aligned}
$$

y

$$
\lim _{n_{1} \ldots n_{k} \rightarrow \infty}\left\|x_{0}+\sum_{i=1}^{k} x_{n_{i}}\right\|=k+1 .
$$

Por ser $E$ un espacio $L w R$ se tiene

$$
\lim _{n \rightarrow \infty}\left\|\tilde{x}_{n}-\tilde{x}_{0}\right\| \leq \lim _{n \rightarrow \infty}\left\|x_{n}-x_{0}\right\|=0 .
$$

Por tanto, hemos demostrado que $E / F$ es un espacio $L k R$.

En forma similar se puede probar el siguiente resultado.

Teorema: 6 Sea $E$ un espacio de Banach reflexivo LwR y $F \subseteq E$ un subespacio cerrado de E. Entonces $E / F$ es un espacio LwR.

Teorema: 7 Sea $E$ un espacio de Banach reflexivo, $U k k$ y $F \subseteq E$ un subespacio cerrado de E. Entonces $E / F$ es un espacio $U k k$.

Prueba: Consideremos $\epsilon>0$ y $\left(\tilde{x}_{n}\right) \subset E / F$ tal que

$$
\left(\tilde{x}_{n}\right) \subset B_{E / F}, \quad \tilde{x}_{n} \stackrel{w}{\rightarrow} \tilde{x} \quad \mathrm{y} \quad \operatorname{Sep}\left(\tilde{x}_{n}\right) \geq \epsilon
$$

Vamos a demostrar que existe un $0<\delta<1$ tal que $\|\tilde{x}\| \leq 1-\delta$. Para cada $\tilde{x}_{n} \in B_{E / F}$ existe

$$
x_{n} \in B_{E} \text { tal que } \pi\left(x_{n}\right)=\tilde{x}_{n} .
$$


Claramente, $\left(x_{n}\right)$ es una sucesión acotada en el espacio reflexivo $E$. Entonces podemos seleccionar una subsucesión $\left(y_{n}\right)$ de $\left(x_{n}\right)$ que converge débilmente a $z \in E$.

Como la norma es débilmente semicontinua inferiormente se tiene que $\|z\| \leq 1$. Pero $\pi\left(y_{n}\right) \stackrel{w}{\rightarrow} \pi(z)$, por lo tanto, $\pi(z)=\tilde{x}$, y así $\|z\| \geq\|\tilde{x}\|=1$, en consecuencia, $\|z\|=1$.

Para $n, m \in \mathbb{N}, \quad n \neq m$ se tiene que

$$
\left\|y_{n}-y_{m}\right\| \geq\left\|\tilde{y}_{n}-\tilde{y}_{m}\right\| \geq \epsilon \quad \Rightarrow \quad \operatorname{Sep}\left(y_{n}\right) \geq \epsilon .
$$

Así, tenemos

$$
\left(y_{n}\right) \subset S_{E}, \quad y_{n} \stackrel{w}{\rightarrow} z \quad \text { y } \quad S e p\left(y_{n}\right) \geq \epsilon .
$$

Por ser el espacio $E \quad U k k$ entonces

$$
\|\tilde{x}\| \leq\|z\| \leq 1-\delta
$$

Así, concluimos que $E / F$ es un espacio $U k k$.

Corolario: 1 Sea $E$ un espacio de Banach $N U C$ y $F \subseteq E$ un subespacio cerrado de $E$. Entonces, E/F es un espacio NUC.

Teorema: 8 Sea $E$ un espacio de Banach reflexivo que satisface la propiedad $(H)$ y $F \subseteq E$ un subespacio cerrado de E. Entonces, E/F tiene la propiedad $(H)$.

Prueba: Sea $\tilde{x} \in S_{E / F}$ y $\left(\tilde{x}_{n}\right) \subset S_{E / F}$ tal que $\tilde{x}_{n} \stackrel{w}{\rightarrow} \tilde{x}$. Queremos probar que $\tilde{x}_{n} \rightarrow \tilde{x}$. En efecto. Existen $x \in S_{E}$ y $x_{n} \in S_{E}, \quad n \in \mathbb{N}$ tales que

$$
\pi(x)=\tilde{x} \quad \mathrm{y} \quad \pi\left(x_{n}\right)=\tilde{x}_{n} .
$$

Claramente, $\left(x_{n}\right)$ es una sucesión acotada y por ser reflexivo el espacio $E$ existe una subsucesión $\left(y_{n}\right)$ de $\left(x_{n}\right)$ tal que $y_{n} \stackrel{w}{\rightarrow} z, \quad z \in E$.

Usando un argumento similar al dado en el teorema 6 se prueba que $\|z\|=1$.

Como $E$ satisface la propiedad $(H)$ se tiene que $y_{n} \rightarrow z$. Pero $\left(y_{n}\right)$ es una subsucesión arbitraria de $\left(x_{n}\right)$ y $\pi\left(y_{n}\right) \rightarrow \pi(z)=\tilde{x}$. Por lo tanto $(\tilde{x}) \rightarrow \tilde{x}$ y as' 1 hemos probado que $E / F$ posee la propiedad $(H)$.

Consideramos conveniente acotar que en [11] aparece el siguiente resultado: un espacio de Banach $E$ posee la propiedad $(D)$ si y sólo si $E$ es un espacio reflexivo que satisface la propiedad $(H)$, definiéndose la propiedad $(D)$ de la siguiente manera, (ver[14]).

Definición 13 Sea E un espacio de Banach. Decimos que E satisface la propiedad $(D)$ si para cualquier conjunto cerrado $C$ disjunto con $B_{E}$ existe $a \in C$ tal que

$$
D\left(a, B_{E}\right) \cap C=\{a\},
$$

donde

$$
D\left(a, B_{E}\right)=C_{0}\left(\{a\} \cup B_{E}\right)
$$

Ahora tenemos el siguiente resultado probado por Montesinos en [11]. 
Corolario: 2 Sea $E$ un espacio de Banach que satisface la propiedad $(D)$ y $F \subseteq E$ un subespacio cerrado E. Entonces, E/F posee la propiedad $(D)$.

En [5] Giles, Sims \& Yorke debilitan la propiedad $(D)$ e introducen la $w$-propiedad $(D)$ en la forma siguiente.

Definición 14 Sea E un espacio de Banach. Se dice que E satisface la w-propiedad $(D)$ si para todo conjunto débilmente secuencialmente cerrado $C$ disjunto de $B_{E}$ existe un $a \in C$ tal que

$$
D\left(a, B_{E}\right) \cap C=\{a\}
$$

En el mismo artículo los autores lograron la siguiente caracterización: un espacio de Banach $E$ posee la $w$-propiedad $(D)$ si y sólo si $E$ es reflexivo.

Finalmente, tenemos el siguiente teorema.

Teorema: 9 Sea $E$ un espacio de Banach que posee la w-propiedad (D) y $F \subseteq E$ es un subespacio cerrado de E. Entonces $E / F$ satisface la w-propiedad $(D)$.

Prueba: Es clara usando la caracterización de la $w$-propiedad $(D)$.

\section{References}

[1] Clarkson, J.A. (1936) "Uniformly Convex Spaces", Trans. A.M.S. 40: 396-414.

[2] Day, M.M. (1973) Normed Linear Spaces, $3^{a}$ edición. Springer-Verlag, New York.

[3] Fan, K.; Glicksberg, I. (1955) "Fully convex normed linear spaces", Proc. Nat. Acad. Scie. USA. 41: 947-953.

[4] Fan, K.; Glicksberg, I. (1958) "Some geometric properties of the spheres in a normed linear space", Duke Math. Jour. 25: 553-568.

[5] Giles, J.R.; Sims, B.; Yorke, A.C. (1990) "On the drop and weakdrop properties for a Banach space", Bull. Aust. Math. Soc. 41: 503-507.

[6] Huff, R. (1980) "Banach spaces which are nearly uniformly convex", Ruck. Mount. Jour. Math. 10: 743-749.

[7] Istratescu, V.I. (s.f.) Strict Convexity and Complex Strict Convexity, Theory and Applications. Marcel Decker, New York.

[8] James, R.C. (1964) "Uniformly non-square Banach space", Ann. Math. 2 80: 542550 .

[9] Lin, B.-L.; Zhang, W. (1991) "Some geometric properties related to uniform convex of Banach spaces, function spaces", Lecture Notes in Pure and Appl.Math. 136, Marcel Decker, New York: 281-294. 
[10] Lovaglia, A.R. (1955) "Locally uniformly convex Banach spaces", Trans. A.M.S. 78: $225-238$.

[11] Montesinos, V. (1987) "Drop property equals reflexivity", Studia Math. 86: 93-100.

[12] Montesinos, V.; Torregrosa, J.R. (s.f.) "Sobre espacios de Banach localmente uniformemente rotundos".

[13] Phelps, R.R. (1960) "Uniqueness of Hanh-Banach extension and unique best approximation", Trans. A.M.S. 95: 238-255.

[14] Rolewicz, S. (1987) "On drop property", Studia Math. 85: 27-35.

[15] Chau-Xun, N.; Jian-Hua, W. (1988) "On the $L k-U R$ and $L-k R$ spaces", Math. Proc. Camb. Phil. Suc. 104: 521-526.

Agradecimiento: mi más sincero agradecimiento al distinguido árbitro por su paciencia en la lectura del trabajo y por sus valiosas recomendaciones. 